\title{
Interpreting Probability
}

\author{
Timothy Childers • Ondrej Majer
}

Published online: 9 March 2012

(C) Springer Science+Business Media B.V. 2012

This special issue of the Journal of Logic, Language and Information contains four selected papers from the 2009 Prague International Colloquium Foundations of Uncertainty: Probability and its Rivals. The contributions in this paper concentrate mainly on probability; we deal with them separately and in turn.

\section{Peter Milne-Probability as a Measure of Information Added}

There have traditionally been two routes to interpreting probability - the quantitative and the qualitative. The dominant tradition has been the quantitative, represented by Dutch Book arguments and decision theoretic restrictions on choices between lotteries. Peter Milne takes the qualitative route. But instead of the usual interpretation of a qualitative ordering over over likeliness, he instead explicates a notion of 'information added'. It soon becomes apparent that there are two different notions of information added that can be extracted from the ordinary use. One is a measure of a new datum's novelty, the other a measure of the possibilities it rules out. Milne shows that the former leads to a peculiar probability-like measure. He uses use well-known (but under-utilized) results to establish that the latter comparative ordering of information added leads to Popper functions.

The interpretation of the functions is, however, "information-theoretic", and not, at least directly, about degrees of belief (or any other conception of probability). It reverses the usual order of proceeding under which measures of information and/or content are taken to be functions of antecedently given probabilities (be they statistical,

T. Childers $(\varangle) \cdot$ O. Majer

Academy of Sciences of the Czech Republic, Prague, Czech Republic

e-mail: timothy.childers@gmail.com

O. Majer

e-mail: majer@flu.cas.cz 
logical, or subjective), and as such, is genuinely novel. As far as we can determine, Milne has provided the first new interpretation of probability in decades. Milne notes possible applications such as justifying the Maximum Entropy Principle, explicating the notion of confirmation and providing semantics for conditionals.

\section{Jeff Paris and Alena Vencovská-Symmetry in Polyadic Inductive Logic}

The logical interpretation of probability, taken as deriving probability assignments to sentences of a language from symmetry constraints, had been left for dead in the early 1970s. Paris and his co-authors have nursed this interpretation back to robust health. The technical innovation is to employ the standard mathematical notion of symmetry as an automorphism. Probability assignments to sentences are given by automorphisms over elements of structures: this provides a precise characterization notoriously lacking in the logical interpretation, and hence clearly delimits the application of symmetry principles thus avoiding the usual 'paradoxes'. It also provides a natural characterization of different symmetry principles.

And yet there was trouble in paradise. Paris and Vencovska 2010 showed that the characterization of symmetry by automorphisms on the structures of a language with unary predicates only leads, in the case of complete ignorance, to a counter-inductive probability measure, Carnap's c- $\uparrow$. This result could be blocked by restricting the class of allowable automorphisms: but the cost of doing so is high, since the naturalness of the characterization of symmetries is lost. Paris and Vencovská conjectured that imposing the requirement that automorphisms on structures on monadic languages be extendable to automorphisms on polyadic extensions of those languages would provide a suitable restriction. This would be a happy result, as it is an entirely natural requirement. Their paper in this journal supports this conjecture. It also represents a marked expansion of the scope and power of inductive logic.

\section{Jacob Rosenthal-Probabilities as Ratios of Ranges in Initial-State Spaces}

Many foundational issues in probability are also foundational issues in statistical mechanics, which is as such a rich mine of ideas for the interpretation of probability. Rosenthal sympathetically surveys a class of ideas for explaining the nature of probabilities arising from deterministic processes. According to this conception, the randomness of statistical mechanical events arises from sensitivity to initial conditions. Since we can never pin down the exact conditions in which an experiment is conducted, we will have a range of possible outcomes, even though the underlying processes are deterministic. Hence experiments give us relative frequencies: the value of these frequencies is determined by the measure of the initial conditions leading to an outcome. The measure is that which is invariant under partitioning of the state-space of initial conditions. Consider the state space of the initial conditions of an outcome: they will, of course, make some proportion of the total initial conditions. If, as we partition the state-space ever more finely, the proportion remains stable, then we can call this measure the probability of the outcome. One way of looking at it is that no matter what set of initial conditions we choose, the relative frequency remains the 
same (the method of arbitrary functions, MAF); another is that within ranges of initial conditions, the proportion of outcomes remains stable (the range conception, RC).

Rosenthal explores the possibility of taking $\mathrm{MAF} / \mathrm{RC}$ as an interpretation of probability, in terms of providing truth conditions. This interpretation is not meant to be universal: it is meant for problems where a state-space of initial conditions with an associated measure can be specified. This concerns statistical mechanics and the classical games of chance in particular, but might also be applied to other fields (Strevens is the main advocate of this view).

However, Rosenthal points out that an RC/MAF interpretation faces a severe problem: probabilities do not remain invariant under redescription. This problem is familiar from logical interpretations of probabilities, particularly in the continuous case. However, there might be more hope in the RC/MAF interpretation since the probabilities arise in physical settings where there would seem to be natural ways of determining the necessary privileged description. Rosenthal shows that the most obvious ways of determining this privileged description cause the RC/MAF interpretation to collapse into other interpretations of probability. He points out a path to avoid this collapse; future research will show if it can be taken.

\section{Alan Hájek}

Conditionals are a perennial philosophical vexation; Alan Hájek aims to show that they are even more vexatious than has recently been thought. A central puzzle in the study of conditionals is their relation to conditional probabilities: they certainly look like they would make a fine match. Yet, the probabilities of conditionals cannot be equated with their corresponding conditional probabilities in any straightforward way. Perhaps, then, there is another more amenable function that would play matchmaker for conditionals and conditional probabilities. Adams proposed equating assertabilities of conditionals with their corresponding conditional probabilities. Adams' Thesis is said to be of paramount importance to understanding reasoning about conditionals.

Yet as Hájek points out, there is a family of such theses, generated by differing scopes of the thesis as well as possible meanings of 'assertability'. His candidate for what most people mean by Adam's Thesis is that the assertability of a conditional is equal to an agent's corresponding conditional probability, where the antecedent has positive probability and assertability is a function of a rational agent's probability function, for all (conditional free) sentential compounds A and B. He identifies two possible weakenings of this thesis; a qualitative version, where assertability is restricted to three values (high, low, middle), and an even more restricted version with just one, high, value.

What sort of function, then, is assertability? It turns out that in order to sustain Adams' Thesis it would have to be a fine-grained function; in fact, finer grained than unconditional probability. Hájek deploys his 'Wallflower' argument, a variant of his generalization of Lewis's first triviality result, to show that the range of the assertability function is richer, has more values, than the unconditional probability function (for finitely-valued probability functions). This is a prima facie most peculiar result which, as Hájek notes, calls for further explanation. Taken together with a number of puzzles about the exact nature of assertability (and cousins like 'assentability'), Hájek 
concludes that the Wallflower argument shows that much work remains to be done if Adam's Thesis is to maintain its privileged position in theorizing about conditionals. However, the qualitative and high assertability versions of the Thesis are not affected by this argument, also pointing the way for future research.

We thank the authors for their papers, and their forebearance during the refereeing process. We also gratefully acknowledge the support of the Grant Agency of the Czech Republic in the form of grant GAP401/10/1504, Formal and Historical Approaches to Epistemology. Finally we thank the Institute of Philosophy, Academy of Sciences of the Czech Republic, as well as our co-organizer Franz Huber of Universität Konstanz for making the conference leading to this issue possible. 